\title{
Relay Optimization for ANC and PNC over Rician Fading Channels
}

\author{
M. A. Jadoon, Z. A. Khan, A. Ahmed, M. Usman, I. Khan, S. Jan \\ University of Engineering and Technology Peshawar, Mardan Campus \\ Khyber Pakhtunkhwa, Pakistan
}

\begin{abstract}
Co-operation and network coding are two such powerful techniques that heightened the proficiency of wireless Networks. This paper mainly focuses on Physical-layer Network Coding (PNC) and Analog Network Coding (ANC), as they are the most studied variants of network coding in literature and represent radically different strategies between themselves, thus shedding light into which problems may hinder the scalability of network coding to more better wireless network topologies. Also, performance of cooperative networks can be enhanced with optimum relay selection. In this paper, we present the simulation results for both Analog Network Coding and Physical-layer Network Coding. We have evaluated the bit error rate (BER) over Rician fading channelsfor ANC and PNC with relay optimization by taking path loss in our system model. BPSK modulation is used for simulation results.
\end{abstract}

\section{Keywords}

Networks, Relay Optimization, Network Coding

\section{INTRODUCTION}

Co-operative relaying has been recently deployed to improve the throughput or reliability of a wireless network. Network Coding (NC), owing to its capability of decently improving the network capacity has attracted the research attention since long, and has given momentum to capacity bounds [1]. Besides, the pronounced and elegant structure offered for network coding, the algebraic NC provides a strong structure to the linear network coding problem [2]. The methodology behind the reference topology $\mathrm{NC}$, can widely be graphed, as there lies an intermediate node that performs combination of linear operations. The data flowing on incoming links are sent to the intermediate node in different time slots sequentially, which is further forwarded to recipients in one time slot, thus reducing the downlink time, that perform such inverse operations to retrieve their own data. This process takes three time slots for successful communication. We refer toit, as digital network coding which is simply XOR operation at intermediate node. But it puts some limitations to the performance by offering an overall wastage of resources, as we can further improve the capacity of cooperative networks [3]-[5].

To overcome such consequences some better approaches like Physical-layer Network Coding (PNC) and Analog Network Coding (ANC) were projected. The effectiveness of NC is preeminently admired owing to its features of, being applicable to all sorts of communication media but, differs in its broadcast nature without affecting the network multiple accesses. Contravention of these two properties owes in hitting the road with the idea of PNC.
Idea of Physical-layer network coding was proposed in [3]. The idea is similar to that of network coding, but at the lower physical layer.The XOR operation is performed at the physical layer by suitable modulation and demodulation. In PNC, the receiver maps simultaneously transmitted signals from two users into the XOR of the two users' data bits. Through a proper modulation-and-demodulation technique at relay nodes, additions of EM signals can be mapped to additions of digital bit streams. The recent research has proved that PNChas provided many potential benefits like increased throughput and reduced energy consumption [4].

ANC was proposed in [5] which open up a whole new research area because of its implications and new design requirements. The approach of analognetwork coding encourages senders to transmit simultaneously, if receivers can leverage all the required information as to reconstruct and disentangle the desired information from the interfered signal. It is usually observed that in communication systems interferences are usually avoided using some special techniques. Here instead of treating interference as a setback to be discouraged, we actually exploit it to improve system's performance. For this, while considering two-way relay network few conditions must be satisfied which are discussed in [4].

The relay combines the signals, Amplify and Forward (AF)scheme is performed on the resultant signal instead of forwarding combined packets as in digital network coding [5]. It is also shown in same paperthat we can double the capacity of 2-way relay network with ANC with less burden of processing. It should be noted that ANC improves the processing speed by increasing intelligence on users to recover the message signal while PNC increases the processing burden at relay user while decoding the signals.

In [6] authors presented SER analysis using PNC over frequency flat fading channel. BER analysis is performed using similar type of work in [3, 4]. In [7], it is shown that amplify and forwardschemes outperformsdecode and forwardscheme in terms of throughput and error probability because of error propagation through the relay.Similar results have been obtained by the authors of [8]. Conventional digital network coding at the packet level has been compared to analog network coding for bidirectional relaying between source pairs in [8].SER analysis is performed over Nakagami$m$ and Rician distributions with different modulation techniques. Closed-form expressions of moment-generating function (MGF) for Space Time Analog Network Coding (STANC) over these fading channels are presented in [9]. ANC is proved to be better network coding in $[10,11]$ in terms of throughput and reliability.

Recently, it has been shown that the performance of wireless relay networks can further be enhanced by properly selecting 
the relays for transmission. Relay selection methods were reported for different schemes. SER performance analysis for optimum relay selection is performed in [12] for decode and forward networks over nakagami- $m$ fading channel. Similar work is done in $[13,14]$ for ANCto select optimum over different fading channels.

We have evaluated the performance of ANC and PNC for optimal relay locations and compared the performance of both techniques in terms of Bit Error Rate (BER). We have derived results for optimum relay location by taking path loss and fading degradationover Rician fading channels.BER is investigated using BPSK modulation. We have proposed the novel concept of relay optimization using network coding techniques and their comparative analysis over Rician fading channels.

The rest of paper is organized as: Section 2 presents the network and communication model. The performance of the proposed system is presented in Section 3 followed by Section 4 , which gives details about simulation results and discussion. Finally, the paper is concluded in Section 5.

\section{SYSTEM MODEL}

We have taken the system model of [15]. Fig 1 illustrates thearrangementof our proposed system model; we have four nodes here, node A (source 1), node B (source 2) and two relay nodes $R_{1}$ and $R_{2}$. Sources $A$ and $B$ send their information signals $x_{1}$ and $x_{2}$ to the relay node $\mathrm{R}_{1}$ and, $x_{3}$ and $x_{4}$ to the relay node $\mathrm{R}_{2}$ respectively. Both relays broadcast the information to nodes $\mathrm{A}$ and $\mathrm{B}$.

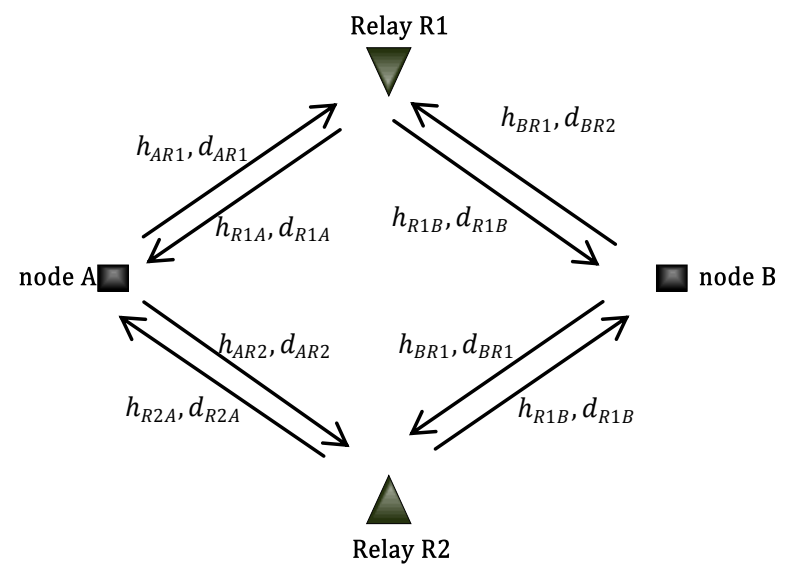

Fig 1: System Model

Communication between terminals takes place in such a way that three nodes are equipped with single antenna and relays work at decode and forward (DF) mode in case of PNC and, amplify and forward (AF) mode in case of ANC.It is assumed that node $\mathrm{A}$ and node $\mathrm{B}$ cannot communicate directly. They could only communicate through relays $R_{1}$ and $R_{2}$. We will evaluate the BER when relay nodes $R_{1}$ and $R_{2}$ will move along their axis to find the optimal relay location.

\subsection{Channel Model}

The basic dilemma that highlighted the divergence amongst theoretical considerations and empirical observations is an issue of perfect signal reception synchronization. It is assumed here that nodes A, B and R, are perfectly synchronized. Sender has no channel state information (CSI) while receiver has perfect CSI. FDMA is used for signals combination at users as both signals to relays $R_{1}$ and $R_{2}$ will have different frequencies. We will consider here identical and independently distributed (i.i.d) Rician Fading channel between three nodes, A, B and R. " $h$ " is the fading magnitude between these nodes. $\alpha=|h|^{2}$ is gamma-distributed random variable. The probability density function of Rician distribution from [15] is given by:

$$
\begin{aligned}
& f(\alpha)=(1+K) \exp (-(1+K) \alpha-K) \\
& \times I_{0}(2 \sqrt{(K(1+K)) \alpha})
\end{aligned}
$$

where $I_{\mathbb{Q}}(\cdot)$ is the zero-th order modified Bessel function of the first kind and Kis the Rician fading factor. For $K=0$, the Rician distribution becomes the Rayleigh distribution.

\subsection{ANC Transmission Protocol}

In case of ANC, again the communication takes place in two time slots but instead of avoiding interference, we exploit the interference.In the first time slot user A and user B transmit their signals $x_{1}$ and $x_{2}$ to the relay simultaneously. Then in the second time slot the interfered signal is broadcasted by relay, $\mathrm{R}$ to both the user in second time slot which is the summation of $x_{1}$ and $x_{2}$. Here amplify and forward operation is performed at relays.

1) $\mathrm{A} \rightarrow \mathrm{R}_{1}$ and $\mathrm{R}_{2}: x_{1}$ and $x_{3}$ and $\mathrm{B} \rightarrow \mathrm{R}_{1}$ and $\mathrm{R}_{2}: x_{2}$ and $x_{4}$ respectively.

$$
\text { 2) } \mathrm{R}_{1} \rightarrow\{\mathrm{A}, \mathrm{B}\}: x_{1}+x_{2} \text { and } \mathrm{R}_{2} \rightarrow\{\mathrm{A}, \mathrm{B}\}: x_{3}+x_{4}
$$

Each user $\mathrm{A}$ and $\mathrm{B}$ extracts its own information by subtracting its own signal from interfered one and combining both signals from both relays using FDMA for better performance.

\subsubsection{Input Output Equations for ANC}

As we know that signal $x_{1}$ and $x_{2}$ are transmitted by node A and $\mathrm{B}$ to $\mathrm{R}_{1}$ respectively, it is also assumed thattransmissions of $x_{1}$ and $x_{2}$ are simultaneous. Again notice that we are considering the path loss, that how distance will effect on signal's power. The received signals at relay $R_{1}$ can be expressed using [4] as:

$$
y_{R 1}=d_{A R 1}{ }^{-\beta} h_{A R 1} x_{1}+d_{B R 1}{ }^{-\beta} h_{B R 1} x_{2}+n_{R 1}
$$

Where $h_{A R 1}$ and $h_{B R 1}$, are Rician fading channel coefficients between $\mathrm{A}$ to $\mathrm{R}_{1}$ and $\mathrm{B}$ to $\mathrm{R}_{1}$, while $d_{p q}$ and $\beta$, are the distance between terminals $p$ - $q$ and path-loss coefficients for these channels, respectively. It is also assumed thatpath-loss coefficient can be perfectly estimated by all of nodes. $n_{\mathrm{R} 1}$ is the complex AWGN at relay $\mathrm{R}_{1}$.

Relay normalizes the received signal and broadcasts the analog network coded signal to users A and B. Both users extract the required information by subtracting their own signal.

\section{Signal at User A:}

The received signal at Relay $R_{1}$ from user $A$ can be written as:

$$
y_{R 1 A}=d_{R 1 A}{ }^{-\beta} h_{R 1 A} y_{R}+n_{1}
$$

As user A knows $x_{1}, h_{A R 1}$, and $d_{\mathrm{AR} 1}$. So it can recover the data as: 


$$
y_{A 1}=y_{R 1 A}-\left|h_{A R 1}\right|^{2}\left|d_{A R 1}{ }^{-\beta}\right|^{2} x_{1}
$$

Where $h_{A R 1}=h_{R 1 A}$. Hence, the recovered signal at use A from relay $\mathrm{R}_{1}$ is:

$$
y_{A 1}=\left(h_{A R 1}\right)\left(h_{B R 1}\right) \times\left(d_{A R 1}^{-\beta}\right)\left(d_{B R 1}^{-\beta}\right) x_{2}+n_{A 1}
$$

Where $n_{\mathrm{A} 1}=h_{A R 1} n_{\mathrm{R}}+n_{1}$. Similarly, we can write the equation of signal from relay $\mathrm{R}_{2}$ :

$$
y_{A 2}=\left(h_{A R 2}\right)\left(h_{B R 2}\right) \times\left(d_{A R 2}^{-\beta}\right)\left(d_{B R 2}^{-\beta}\right) x_{4}+n_{A 2}
$$

User A will combine both the signals $y_{A 1}$ and $y_{A 2}$ to recover its information

\section{Signal at User B:}

Similarly received signal at Relay from user B is:

$$
y_{R 1 B}=h_{R 1 B} d_{R 1 B}^{-\beta} y_{R}+n_{2}
$$

And user B knows $x_{2}$ and $h_{B R 1}, d_{\mathrm{BR} 1}$. Similarly it can recover the data as:

$$
y_{B 1}=y_{R 1 B}-\left|h_{B R 1}\right|^{2}\left|d_{B R 1}{ }^{-\beta}\right|^{2} x_{2}
$$

Where $h_{B R 1}=h_{R 1 B}$.

Hence, the recovered signal at user $B$ can be written as:

$$
y_{B 1}=\left(h_{A R 1}\right)\left(h_{B R 1}\right) \times\left(d_{A R 1}^{-\beta}\right)\left(d_{B R 1}^{-\beta}\right) x_{1}+n_{B 1}
$$

Where $n_{\mathrm{B} 1}=h_{B R} n_{\mathrm{R}}+n_{2}$. Similarly, we can write the equation of signal from relay $\mathrm{R}_{2}$ :

$$
y_{B 2}=\left(h_{A R 2}\right)\left(h_{B R 2}\right) \times\left(d_{A R 2}^{-\beta}\right)\left(d_{B R 2}^{-\beta}\right) x_{1}+n_{B 2}
$$

User B will combine both the signals $y_{B 1}$ and $y_{B 2}$ to recover its information

\subsection{PNC Transmission Protocol}

For PNC protocol, performance criterion can be demonstrated in such a way the whole communication concludes in two time slots as in the first time slot user A and user B transmit their signals $x_{1}$ and $x_{2}$ to the relay simultaneously. Decode and Forward operation is performed at relay node $\mathrm{R}$ in this case by suitable modulation and demodulation. Relay $\mathrm{R}$ decodes the signals $x_{1}$ and $x_{2}$, and then forwards the summation of two signals. This can be summarized as follow:

1) $\mathrm{A} \rightarrow \mathrm{R}_{1}$ and $\mathrm{R}_{2}: x_{1}$ and $x_{3}$ and $\mathrm{B} \rightarrow \mathrm{R}_{1}$ and $\mathrm{R}_{2}: x_{2}$ and $x_{4}$ respectively.

2) $\mathrm{R}_{1} \rightarrow\{\mathrm{A}, \mathrm{B}\}: x_{1} \oplus x_{2}$ and $\mathrm{R}_{2} \rightarrow\{\mathrm{A}, \mathrm{B}\}: x_{3} \oplus x_{4}$

Each user $\mathrm{A}$ and $\mathrm{B}$ extracts its own information by again XORing its own signal with signal forwarded by Relay node $\mathrm{R}_{1}$ and $\mathrm{R}_{2}$. $\oplus$ shows the XOR operation performed at physical-layer at relay nodes. We are using BPSK and QPSK for our proposed system model.

\subsubsection{Input Output Equations for PNC}

Input and output equations for PNC transmission protocol are not different from the case of ANC instead we will remove the effect of fading coefficients $h_{A R 1}$ and $h_{B R 1}$ and, $h_{A R 2}$ and $h_{B R 2}$ at relay nodes $\mathrm{R}_{1}$ and $\mathrm{R}_{2}$ by performing the decode operation. Also, again XOR operation is performed at users with their own signal after demodulation to recover the original signals. So the signals at node $A$ from relays $R_{1}$ and $\mathrm{R}_{2}$ are as follows:

$$
\begin{gathered}
y_{A 1}=\left(h_{A R 1}\right) \times\left(d_{A R 1}{ }^{-\beta}\right) x+n_{A 1} \\
y_{A 2}=\left(h_{A R 2}\right) \times\left(d_{B R 2}{ }^{-\beta}\right) x^{\prime}+n_{A 2}
\end{gathered}
$$

Where $x=x_{1} \oplus x_{2}$ and $x^{\prime}=x_{1} \oplus x_{2}$.

Both signals $y_{A 1}$ and $y_{A 2}$ will be added and XORed with original signal to recover the information. Similarly we can write the equations for user B.

\section{PERFORMANCE ANALYSIS}

The bit error probability is assumed as an important consideration for basic performance measurements. The aspiration of this paper is specifically to calculate the Bit Error Rate (BER) for broadband Analogue Network Coding over Rician fading channel and its intelligent variant physicallayer network coding. That will substantially reproduce their comparative in-depth. Relay locality is considered as a focusing factor for discussion along comparative results. We will move the relay along the axis to present the idea of optimal relay location in ANC transmission protocol and will compare its results with PNC. Another highlighted parameter is of $K$, for different values of $K$ that is for $K=0, K=1$ and $\mathrm{K}=4$, analysis are made discretely. While in this paper their combined effect is demonstrated.

It is to be noted that the main objective of this letter is not to revisit path-selection algorithms, but to focus on giving an exact analysis of selective-relaying schemes that is valid for allSNR regimes.But the concept is given to justify the different network coding techniques in relay selection that how these techniques will affect the performance of network in this scenario.

\subsection{Simulation Environment}

Simulation model is shown in the figure 2 . It is assumed that users A and B cannot communicate directly. Relays are intermediarynodes that complement the transmission. The performances for both ANC and PNC are recorded sequentially. Results are evaluated for sub-urban area by taking the value of path-loss exponent $\beta=3$; while $(d)^{-c}$ is total path loss. Total distance between users A and B is taken as $d_{o}$ and is normalized to 1 for simplicity in simulations. All distances are normalized to $d_{o}$. We will keep the distances of both nodes $\mathrm{A}$ and $\mathrm{B}$ at same distance from relay nodes $\mathrm{R}_{1}$ and $R_{2}$, and then we will move relays along their axes at different positions from both nodes. For simplicity, results are only shown for node A as due to symmetry; performances of both nodes A and B are similar.

Node A is located at coordinates $(0,0)$ and the node B at $\left(0, d_{o}\right)$ within a square of side length of $d_{o}$, while the two relays are located between nodeA and B as shown in Fig.2. The distances between relays are set to be $d_{o} / 2$. Thus the $y$ axis of the relay 1 and 2 are set to be equal to $d_{o} / 4$ and $-d_{o} /$ 4 , respectively, as shown in simulation model figure 2 . We will take different combinations for optimum location of both relays.

\subsection{BER Analysis}

For different possible projections of both relays, we will initially evaluate the BER by taking the value of $\mathrm{SNR}=8$. For this value of SNR an optimum location is evaluated for both ANC and PNC in effect of least BER. Again note that we 
have taken different possible combinations of both relay locations and evaluated the BER along with distances of both users $\mathrm{A}$ and $\mathrm{B}$ as shown in figure 3 and figure 4 .

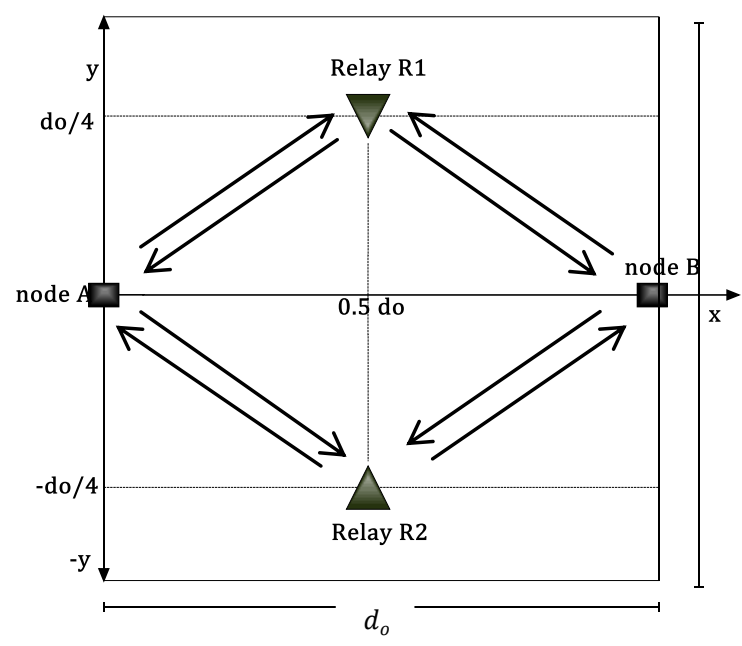

Fig 2: Simulation Model

\subsubsection{BER Comparison of ANC and PNC}

Next we have presented results of BER versus SNR for the comparative analysis of both ANC and PNC, in figure 5. Here results are obtained for different values of Rician parameter $\mathrm{K}$ which are $\mathrm{K}=0,1,4$.

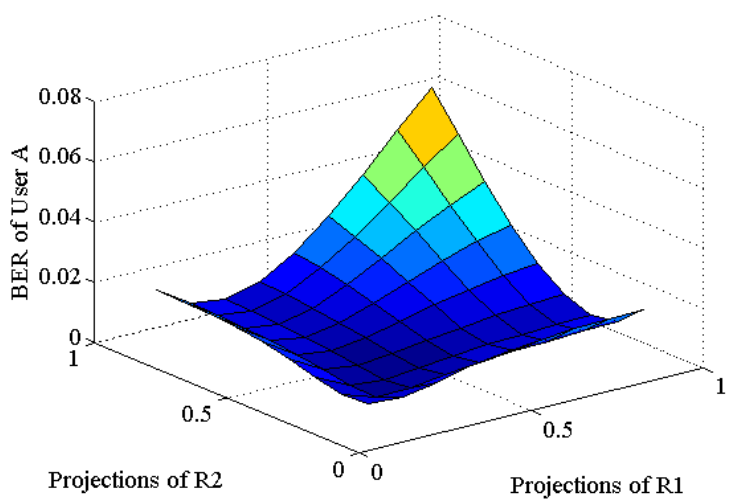

Fig 3: 3-Dimensions graph showing BER for different projections of both relays at $\mathrm{SNR}=8$ using $\mathrm{ANC}$ protocol.

The analysis is made for the optimum location when relay $R_{1}$ is at $0.75 d_{o}$ and relay $\mathrm{R}_{2}$ is at $0.25 d_{o}$. Note: For $\mathrm{K}=0$, Rician distribution becomes Rayleigh distribution.

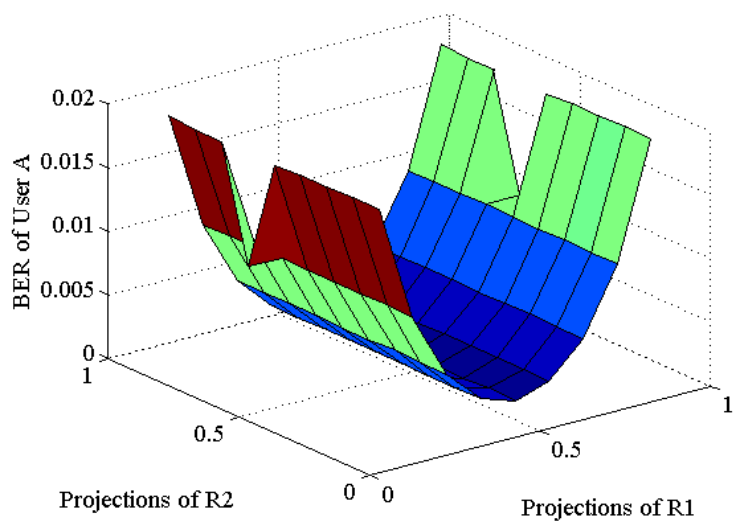

Fig 4: 3-Dimensions graph showing BER for different projections of both relays at $\mathrm{SNR}=8$ using PNC protocol.

\subsection{Cooperation Gain}

Here in this section we have compared the cooperation BER using network coding and direct component BER and evaluated the gain.

$$
\text { Gain }=B E R_{d} / B E R_{c}
$$

Where $B E R_{d}$ and $B E R_{c}$ are bit error rates of direct transmission and BER with cooperation, when relays are employed, respectively.Figure 6 and 7 shows the gain analysis for ANC and PNC along with different projections of both relays. This gain will help us to understand easily the concept of cooperation with different network coding schemes in terms of BER. For this system model we have taken different locations for optimum relay selection, evaluated BER for all locations for the same SNR $=8$ using both ANC and PNC, and compared all these values of BER with the BER of direct component. These evaluations are also done over the Rician fading channels.

\section{RESULTS AND DISCUSSION}

This sub-section formally discusses the graphical results attained here. The results are obtained from Monte Carlo simulations. From simulation results we have deduced some conclusions about the performance of two-way relay network for Analog Network coding (ANC) and physical-layer network coding (PNC) using Rician fading channel. We assumed that channel coefficients remain constant during transmission. Also, receiver has perfect channel estimation while transmitter does not have any in terms of CSI. Proceeding with the ensuing work, the projection of various distances for both the relays are encountered, whose graphical aspect was analyzed along their BER results. This result was supplementarydiscussed taking in an account of a constantSNR $=8$, for which an optimum relay location was designated having best performance showing least BER. For this premium location a judgment was set that figured out the comparison along most important network topologies discussed above that is ANC and its variant counterpart PNC. 


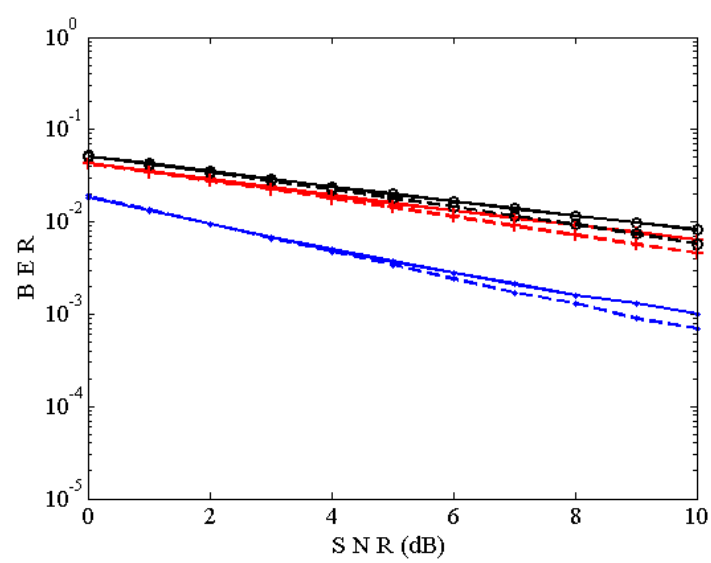

Fig 5: BER comparative analysis of ANC and PNC over Rician Fading channel for $K=0,1$, and 4 .

This whole pictogram was extended with inclusion of various SNR values despite of single SNR in addition to their BER, a productive analysis was outlined amongst ANC and PNC that generated a rack of comparative information.

From graphical perspective we deduced some results particularly from figure 3 and figure 4 . Figure 3 was attained by changing relative position of both the relays for ANC investigation, while figure 4 contained similar upshots for PNC.

We examined minimum BER for the relays aligned at center as shown in figure 2. This demonstrates preeminent system performance, which gradually got declined with the changing position of relays towards the edges.

In figure 3 results were attained similarly for PNC but generated some diverse results. Seconding to the previous results it also showed better performance for both of the relays aligned exactly at the center as in figure 2 , but reduced the performance by drastically increasing the BER

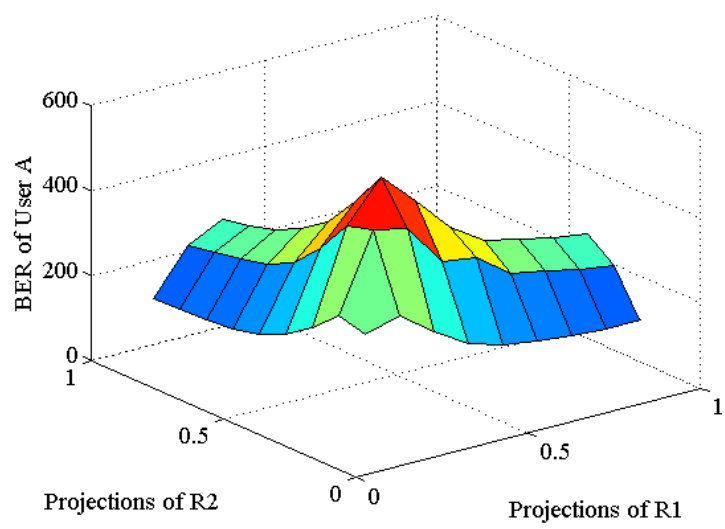

Fig 6: Cooperation gain for ANC

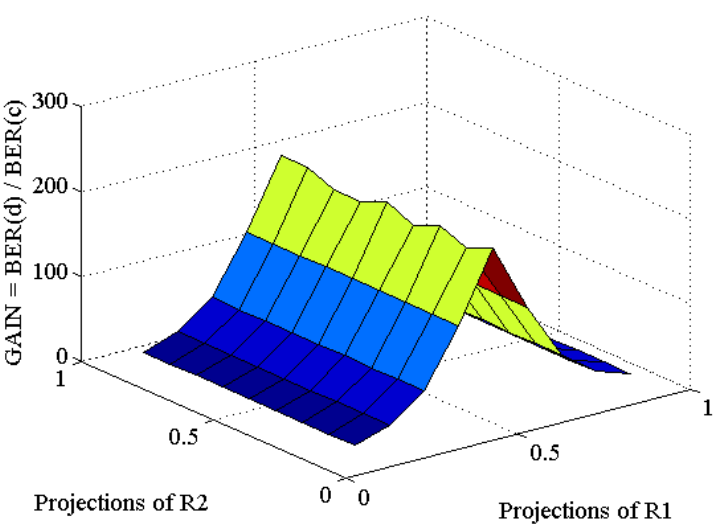

Fig 7: Cooperation gain for PNC

This shows contradiction with previous ANC results where performance degradation with diverting relay location showed a smooth variation by increasing the BER smoothly, While, this anomalous approach was not encountered for PNC in graph 4 . From this we can infer that PNC strictly demand an optimum relay location for better performance that is in contrast to ANC. In figure 5, we have presented the analysis of both network coding techniques along with Rician K parameter and for the optimum location. It is observed that PNC has shown slightly good results as compared to ANC. This is because PNC is more reliable due to intelligence at relay nodes. Also, it is observed that BER decreases by increasing the value of $\mathrm{K}$ factor from 0 .

These results were preceded ahead in figure 6 and 7 for ANC and PNC respectively, which complemented the cooperation gain along projection of both relays. We analyzed, that in existing scenarios cooperative relays produced better performance as compared to the direct transmission in terms of BER.

\section{CONCLUSION}

In this paper, a new extension and approach is proposed for comparative analysis of analog network coding and physical layer network coding over Rician fading channel for optimum relay allocation. Elementary aspiration of this letter is to give a comparative in-let to both the important strategies with regard to their BER estimation. The important results are discussed for various presumed scenarios, mainly considering relay manipulations by changing their respective placements. Some useful results were deduced that added more to the arena of network coding. We have seen that analog network coding has decreased the system processing and gave the better performance over two way-relay network. Also, gain analysis has shown us the advantage of cooperation in terms of BER. Our results open up new questions for better wireless network deployment. This work can be further extended with multiple relays and users, where assumptions we have made should be removed to make the system more practical. Mathematical modeling of this network is left for future work. 


\section{REFERENCE}

[1] S. Y. R. Li R. W. Yeung R. Ahlswede, N. Cai. Network information flow.IEEE Trans. Inform. Theory, 46:12041216, 2000.

[2] T. Ho, D.S. Lun, "Network Coding," Cambridge University Press, New York, USA, 2008.

[3] S. Zhang, S. C. Liew, and P. P. Lam, "Hot topic: physical-layer network coding," in Proceedings of the 12th AnnualInternational Conference onMobile Computing and Networking(MOBICOM '06), pp. 358365, Los Angeles, Calif, USA, September 2006.

[4] S. Zhang, S. C. Liew, and L. Lu, "Physical layer network coding schemes over finite and infinite fields," in Proceedingsof IEEE Global Telecommunications Conference (GLOBECOM'08), pp. 3784-3789, New Orleans, La, USA, November-December 2008.

[5] S. Katti, S. Gollakota, and D. Katabi, "Embracing wireless interference: analog network coding," in Proceeding of ACMConference on Computer Communications (SIGCOMM '07), pp. 397-408, Kyoto, Japan, August 2007.

[6] Lu, K,Shengli Fu, Yi Qian, Hsiao-Hwa Chen, "SER Performance Analysis for Physical Layer Network Coding over AWGN Channels,"IEEE GLOBECOM, Honolulu, HI, Nov 30, 2009-Dec 4, 2009.

[7] Riemensberger, M. Sagduyu, Y.E. Honig, M.L. Utschick, W."Comparison of Analog and Digital Relay Methods with Network Coding for Wireless Multicast." Communications, 2009.ICC '09. IEEE International Conference on Digital Object Identifier, 2009 , Pages: 1 5

[8] Sagduyu, Y.E. DongningGuo Berry, R."On the Delay and Throughput of Digital and Analog Network Coding for Wireless Broadcast." Information Sciences and Systems, 2008.CISS $2008.42^{\text {nd }}$ AnnualConferenceonDigital Object Identifier, 2008 , Page(s): 534 - 539

[9] Shujaat Ali Khan Tanoli, Imran Khan, NandanaRajatheva, and Fumiyuki Adachi, "Advances in Relay Networks: Performance and Capacity Analysis of Space-Time Analog Network Coding." EURASIPJournal on Wireless Communications and Networking. Volume 2010, April 2010.

[10] Sushant Sharma, Yi Shi, Jia Liu, Y. Thomas Hou, SastryKompella"Is Network Coding Always Good for Cooperative Communications?"INFOCOM, 2010 Proceedings IEEE, Page(s): 1 - 9

[11] P. Popovski and H. Yomo, "Wireless Network Coding by Amplify-and- Forward for Bi-Directional Traffic Flows," IEEE Commun. Lett., vol. 11, no. 1, pp. 16-18, Jan. 2007.

[12] ChunxiaoCai, YuemingCai, Junquan Hu, Weiwei Yang, Wendong Yang,"Performance Analysis of Selection Decode-and Forward Relay Networks overnakagami-m fading channels." 12 th IEEE International Conference on Communication Technology (ICCT), 2010, Nanjing, china.

[13] Y. Zhao, R. Adve and T. J. Lim. "Symbol error rate of selection amplify and forward relay systems."IEEE Communications Letters, vol. 10, No.11, pp. 757-759, Nov. 2006.

[14] S. A. Fares, F. Adachi and E. Kudoh. "Novel Cooperative Relaying Network Scheme with Exchange Communication and Distributed Transmit Beamforming" 5th IEEE VTS Asia Pacific Wireless Communications Symposium (APWCS 2008), Sendai, Japan, August 21-22,2008

[15] M. K. Simon and M.-S. Alouini, "Digital Communication over Fading Channels", John Wiley \& Sons, New York, NY, USA,2005. 